\title{
Implementing Human Papillomavirus Testing in a Public Health Hospital: Challenges and Opportunities
}

\author{
Mariana Carmezim Beldi ${ }^{a}$ Maricy Tacla ${ }^{a}$ Helio Caiaffa-Filho ${ }^{c}$ \\ Alexandre Ab'saber ${ }^{b}$ Sheila Siqueira ${ }^{b}$ Edmund Chada Baracat ${ }^{a}$ \\ Venancio Avancini Ferreira Alves ${ }^{b, d}$ Adhemar Longatto-Filho ${ }^{d, e}$
}

C) S. Karger AG, Basel

PROOF Copy
for personal
use only

ANY DISTRIBUTION OF THIS

ARTICLE WITHOUT WRITEN

CONSENT FROM S. KARGER

AG, BASEL IS A VIOLATION

OF THE COPYRIGHT.

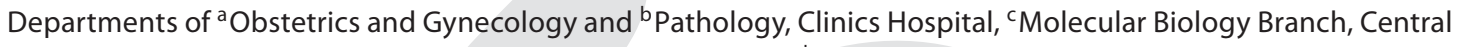
Laboratory Division, Clinics Hospital, School of Medicine, and d Laboratory of Medical Investigation 14, Department of Pathology, Medical School, University of São Paulo, São Paulo, Brazil; e Life and Health Sciences Research Institute, School of Health Sciences, University of Minho, and PT Government Associate Laboratory, Braga, Portugal

\section{Key Words}

Cytology $\cdot$ Pap test $\cdot$ Human papillomavirus testing $\cdot$ Primary screening

\begin{abstract}
Objectives: Robust evidence now supports human papillomavirus (HPV) testing as a more effective option to screening and as more sensitive than cytology in detecting highgrade cervical intraepithelial neoplasia . Our goal was to analyze the performance of the Hybrid Capture II (HC2) assay for high-risk HPV (hrHPV) in women undergoing gynecological examination at a public health hospital as part of the evaluation of HPV screening as an alternative or complement to cytology. Study Design: This analysis is a subset of a cross-sectional study carried out at a large public hospital serving a predominantly low-resource population. A total of 705 women were enrolled; the sensitivity and specificity of each test were estimated and compared. Results: The analysis identified 272 hrHPV-positive women (mean age 36.3 years) and 433 hrHPV-negative women (mean age 41.2 years). HPV testing showed a significantly increased sensitivity of
\end{abstract}

\section{KARGER \\ Fax +41 613061234 E-Mail karger@karger.ch} www.karger.com
(C) 2012 S. Karger AG, Basel

$0001-5547 / 12 / 0000-0000 / \$ 38.00 / 0$

Accessible online at:

www.karger.com/acy the $\mathrm{HC} 2$ assay versus cytology ( 84.5 vs. $69.7 \%$; $\mathrm{p}<0.0001)$ but a lower specificity (49.90 vs. $88.78 \%$; $p<0.0001$ ). Conclusion: The combination of both methods seems to be useful in improving detection of cervical lesions.

Copyright $\odot 2012$ S. Karger AG, Basel

\section{Background}

Cervical cancer is the second most common cancer in women worldwide [1], although its incidence has fallen by $50 \%$ or more in developed countries since the introduction of Pap smear screening. However, a single cervical cytology test is insensitive for the detection of precancer and cancer of the cervix [2]. Human papillomavirus (HPV) infection is very common in young women after the onset of sexual activity and, when it persists, the viral oncoproteins can disrupt cell-cycle controls, resulting in cervical intraepitheal neoplasia (CIN). In their mildest appearance, CIN1, these lesions are generally no more than manifestations of HPV infection, but at their most severe, CIN3, the risk of progression to invasive

Correspondence to: Dr. Adhemar Longatto-Filho

Laboratory of Medical Investigation (LIM) 14

Faculty of Medicine, University of São Paulo

1246-903 São Paulo (Brazil)

Tel. +55 113061 7413, E-Mail longatto16@ hotmail.com 
cancer is higher. Fortunately, this transition usually takes years or decades, allowing for the opportunity to detect the cancer by cytology [3]. A persistent infection with high-risk (hr)HPVs is the key event in the development of cervical cancer and its precursor lesions; consequently, the efficacy of cervical cancer screening can be improved by HPV testing. Substantial evidence now shows that HPV testing is more sensitive than cytology in detecting high-grade CIN, and some randomized controlled trials have demonstrated that it can detect persistent - and therefore clinically relevant - neoplasia earlier than cytology can [4]. However, HPV testing is less specific than cytology, most likely because of the substantial number of HPV-positive women who harbor transient infections that clear spontaneously and do not cause high-grade lesions.

One HPV test, the Hybrid Capture 2 (HC2; Digene Corporation, currently QIAGEN, Gaithersburg, Md., USA) has been approved by the US Food and Drug Administration (FDA) for use in cervical cancer screening for the triage of equivocal cytology and atypical squamous cells of undetermined significance (ASCUS), and as a screening adjunct to cytology in women older than 30 years [5]. Given the evidence favoring the use of a combination of cytology and HPV testing, we sought to investigate different cervical cancer screening strategies to ascertain the presence of cervical HPV-induced lesions, such as HPV testing, conventional Pap smear cytology and colposcopy, in the Hospital das Clínicas, School of Medicine of São Paulo State University, a reference public health hospital in Brazil. The objective was not to verify the possibility of introducing $\mathrm{HC} 2$ as an alternative method for screening a large general population, but to evaluate it as a methodological option for optimizing the screening of women undergoing gynecological examination for a variety of clinical reasons.

\section{Materials and Methods}

\section{Study Population}

Between August 2008 and July 2009, 705 women were referred for a gynecology examination for different reasons (e.g., previous abnormal Pap test, follow-up of a treated cervical lesion). Eligibility requirements for participation in the study were as follows: age 17-81 years and not currently pregnant. Women with chronic immune suppression and those who were undergoing treatment for a CIN were evaluated in different groups. All women were invited to participate in the study and were enroled if they provided written informed consent. The study was approved by the University of Sao Paulo ethics committee.

\section{Clinical Examinations}

At the first visit, cytological samples were taken immediately after an HPV DNA test. For the conventional cytological test, an Ayre's spatula was used to collect cells from the transformation zone, and a cytobrush was used to collect cells from the endocervical canal. A cervical specimen for HPV DNA testing was taken by rotating a Dacron-tipped swab in the endocervical canal and swabbing it on the ectocervical epithelium. The swab was then placed in a specially prepared specimen transport medium (Digene Corp.).

All women underwent colposcopy at the first visit, which was performed by members of a team of gynecologists with extensive training. Colposcopically guided biopsies of the worst visible lesions were obtained. Women with histological diagnoses of CIN2-3 were referred for loop electrosurgical excision procedures. Women with invasive cancer were referred to a gynecologic oncologist for appropriate staging and treatment. When the detected lesion did not correlate with cytological results, diagnostic loop electrosurgical excision procedures were performed.

\section{Cytological and Histological Evaluation}

For conventional cytology, a thin-layer slide was stained with Papanicolaou stain, screened by a cytotechnologist and reviewed by pathologists at the Hospital das Clínicas Medical Center (who had no knowledge of the other laboratory or clinical data). Cellular changes were classified according to the Bethesda system. Biopsy specimen slides were reviewed by a pathologist with no knowledge of other clinical or laboratory data.

\section{HPV DNA Testing}

HPV testing was done using HC2 (Digene Corp.) with only the high-risk group of probes. These probes are designed to detect HPV types $16,18,31,33,35,39,45,51,52,56,58,59$ and 68 . These tests were done according to the manufacturer's instructions and results given as the ratio of the relative light units, which was considered positive when a relative light unit was greater than 1.0. Before initiating the study, the laboratory had completed the manufacturer's proficiency test and obtained very similar results on test samples. The HC2 manufacturer had no involvement in this study, and all supplies and reagents were purchased at the regular cost.

\section{Statistical Analysis}

Sensitivity, specificity and positive and negative predictive values were calculated, generating $2 \times 2$ tables, cross-classifying screening results and biopsy results. All estimates were calculated using biopsy-proven CIN2+ and CIN $3+$ as endpoints. The relative sensitivity of HPV testing using HC2 versus conventional cytology was calculated as the ratio of detection rates of CIN2+ in the HPV-positive and HPV-negative groups, and we analyzed women according to age groups (15-20, 21-25, 26-29 and 30 years or older). Because the prevalence of HPV infection is higher in younger women, our strategy was to compare relative sensitivity in each age group. Significance was defined as $p<0.05$. Comparisons between proportions were made with the $\chi^{2}$ test, and $95 \%$ confidence intervals (CIs) for corrected estimates were calculated with the use of bootstrap resampling in which the sampling fractions were considered fixed. All analyses were conducted using SPSS software (version 15.0, SPSS Inc., Chicago, Ill., USA). 
Table 1. Distribution of HC2-positive test results for cytological results and age

\begin{tabular}{|c|c|c|c|c|c|c|c|c|}
\hline \multirow[t]{3}{*}{ Cytological results } & \multicolumn{8}{|c|}{ Patient ages distributed by intervals } \\
\hline & \multicolumn{2}{|c|}{$15-20$ years } & \multicolumn{2}{|c|}{$21-25$ years } & \multicolumn{2}{|c|}{$26-29$ years } & \multicolumn{2}{|c|}{$\geq 30$ years } \\
\hline & $\mathrm{HC} 2+$ & $\mathrm{HC} 2-$ & $\mathrm{HC} 2+$ & HC2- & $\mathrm{HC} 2+$ & $\mathrm{HC} 2-$ & $\mathrm{HC} 2+$ & $\mathrm{HC} 2-$ \\
\hline HSIL & 1 & - & 2 & - & 7 & 1 & 40 & 6 \\
\hline LSIL & 2 & 3 & 12 & 2 & 19 & - & 56 & 11 \\
\hline ASCUS & - & - & - & 4 & 3 & 2 & 14 & 15 \\
\hline AGC & - & - & - & - & 2 & - & 4 & - \\
\hline AIS & - & - & - & - & - & - & 1 & - \\
\hline Invasive cancer & - & - & - & - & - & - & 1 & - \\
\hline Negative & 8 & 11 & 15 & 39 & 17 & 34 & 68 & 303 \\
\hline
\end{tabular}

\section{Results}

A total of 705 women were recruited. They were divided into 2 large groups: HPV-positive $(\mathrm{n}=272)$ and HPV-negative $(\mathrm{n}=432)$. In the HPV-positive group, the median age was 36.3 versus 41.2 years in the negative group; $38.6 \%$ of the women had oncogenic HPV types, detected by HC2. Screening with the Pap test identified 168 (61.8\%) women with cytological abnormalities in the HPV-positive group. The HPV-negative group had 44 (10.2\%) abnormal smears. Both groups were divided into 4 prespecified age groups: 17-20, 21-25, 25-29 and older than 30 years (table 1). Women aged 30 years or more had a higher prevalence of oncogenic HPV than younger women (25 vs. $13.6 \%$, respectively), but were more likely to have normal cytological results (36 vs. 15\%). Women with more severe cytological diagnoses were more likely to have a positive $\mathrm{HC} 2$ test than women with less severe cytological lesions.

Histopathological results were available for 239 women who underwent colposcopically guided biopsy. Table 2 summarizes the results.

Overall, testing with the HC2 test resulted in a significantly increased sensitivity when compared to the cytological method (84.5 vs. 69.7; p < 0.0001; CI 95\%), but it was less specific (49.90 vs. 88.78\%; p <0.0001; CI 95\%). We also identified more CIN2+ and CIN3+ in women aged 30 years or older than in the rest of the other groups. Sensitivity and specificity did not differ significantly between cytology and HC2 testing in the 15-29-year age group (table 3 ).

The combination of Pap smear and HPV DNA testing resulted in a sensitivity of $90.91 \%$ and a specificity of $48.04 \%$ for CIN2+ and CIN3+, indicating that using the two simultaneously improved sensitivity but not specificity.
Table 2. Distribution of HC2-positive test results with histological findings for 239 biopsies, diagnoses and age

\begin{tabular}{|c|c|c|c|c|c|c|c|c|}
\hline \multirow{2}{*}{$\begin{array}{l}\text { Histological } \\
\text { results }\end{array}$} & \multicolumn{2}{|c|}{$15-20$ years } & \multicolumn{2}{|c|}{$21-25$ years } & \multicolumn{2}{|c|}{$26-29$ years } & \multicolumn{2}{|c|}{$\geq 30$ years } \\
\hline & $\mathrm{HC} 2+$ & $\mathrm{HC} 2-$ & $\mathrm{HC}+$ & $\mathrm{HC} 2-$ & HC2+ & $\mathrm{HC} 2-$ & $\mathrm{HC} 2+$ & HC2- \\
\hline Negative & 5 & 9 & 11 & 9 & 15 & 6 & 34 & 73 \\
\hline CIN1 & 1 & - & 6 & 1 & 7 & - & 16 & 2 \\
\hline CIN2 & 1 & - & - & - & 2 & - & 8 & 1 \\
\hline CIN3 & 1 & - & - & - & 3 & 1 & 12 & 1 \\
\hline VAIN 1 & - & - & - & - & 2 & - & 2 & - \\
\hline VAIN 2 & - & - & - & - & - & - & - & 2 \\
\hline VAIN 3 & - & - & - & - & 2 & - & 1 & - \\
\hline VIN 2-3 & - & - & 2 & - & - & - & 1 & 1 \\
\hline MIC & - & - & - & - & - & - & 1 & - \\
\hline
\end{tabular}

Table 3. Distribution of sensitivity and specificity of HC2 by age as compared with cytological (HSIL endpoint) results and age

\begin{tabular}{lll}
\hline Endpoint CIN2-3 & Sensitivity & Specificity \\
\hline $15-20$ years & $100 \%, \mathrm{p}=0.26$ & $61 \%, \mathrm{p}=0.26$ \\
$21-25$ years & $100 \%, \mathrm{p}=0.5$ & $56 \%, \mathrm{p}=0.5$ \\
$26-29$ years & $83 \%, \mathrm{p}=0.7$ & $33 \%, \mathrm{p}=0.7$ \\
$\geq 30$ years & $91 \%, \mathrm{p}<0.0001$ & $70 \%, \mathrm{p}<0.0001$ \\
\hline
\end{tabular}

\section{Discussion}

Our results agree with those of previous reports that have demonstrated the efficiency of molecular tests in recognizing $\mathrm{HPV}$ infection and its correlated lesions. HC2 was clearly superior to cytology in screening women with HPV-induced lesions, with a significantly higher 
sensitivity. The introduction of a molecular method into the hospital routine is also important for validating a new screening paradigm in the context of cervical carcinogenesis [2].

The recognition of the strong causal relationship between persistent infection of the genital tract with hrHPV types and the occurrence of cervical cancer has resulted in the development of a number of DNA and RNA detection systems for screening, to complement the limitations of cytology [3]. In the USA, for example, HPV testing with cytology is approved for primary screening of women aged 30 years and older.

Several studies based on double testing to screen for cervical lesions have shown that HPV testing has greater sensitivity than cytology but lower specificity in detecting high-grade CIN [6]. We also observed that HPV testing has a higher sensitivity but lower specificity. Recent work, assessing absolute cross-sectional accuracy, found that the overall sensitivity of $\mathrm{HC} 2$ as a primary screening tool for detecting underlying high-grade CIN is $89.7 \%$ (range 50-100\%) and that the pooled specificity of HC2, excluding high-grade cervical precancer, is $88.2 \%$ (range 61-95\%) [3]. Furthermore, HC2 sensitivity has been consistently high in 8 studies conducted in Europe and North America, but in developing countries such as Brazil, Zimbabwe and South Africa, the sensitivity for HC2 is lower $(83,81$, and $88 \%)$.

Several factors may influence this lower sensitivity, such as sample contamination by acetic acid or Lugol's iodine or deterioration of the sample because of exposure to high environmental temperatures. The $\mathrm{HC} 2$ protocol notes that HC2 samples should be collected before other procedures. The lack of inclusion of other oncogenic HPV types in the HC2 probe cocktail is another potential explanation for the lower sensitivity of the HC2 assay for some series, but this assumption is debatable [3]. On the other hand, the pooled specificity of $\mathrm{HC} 2$ can be $6 \%$ lower than that of cytology, but the combination of cytology with HC2 yields higher values than cytology alone (46 vs. 35\%); adding Pap smears to the $\mathrm{HC} 2$ test along with considering ASCUS or worse as a positive cytological result also increases sensitivity by 6 and 4\%, with CIN2 and CIN3 as endpoints, respectively. As a consequence, the specificity decreases by 5 and $7 \%$. Accordingly, the most useful role for cytology may be its application, in women whose primary screen yielded positive results for HPV DNA, to avoid referral and overtreatment of those women with minimal or undetectable cytological abnormalities [3].

Cross-reactivity may play a role in $\mathrm{HC} 2$ specificity. Previous studies have suggested that HC2 may cross-re- act with HPV types either not associated with, or having undetermined associations with cancer (nononcogenic HPV), such as 6, 11, 26, 40, 42, 53, 66, 67, 71, 83 and 84 . Castle et al. [2] evaluated the effects of cross-reactivity on clinical performance by calculating sensitivity and specificity with and without cross-reactivity for the detection of high-grade cervical lesions. They tested 954 specimens using HC2 and HPV type-specific MY09/MY11 L1 consensus primer PCR. According to their results, clinical sensitivity increased from 84.3 (without cross-reactivity) to $87.9 \%$, specificity decreased from 89.6 to $88.1 \%$, and referral rates increased from 11.7 to $13.2 \%$ for the detection of CIN2+. They also found that the clinical effect of cross-reactivity varied by cytological interpretation. Among women with normal cytology, cross-reactivity significantly improved the accuracy of identifying CIN2+. However, among women with equivocal or mildly abnormal cytological alterations, cross-reactivity decreased the accuracy of HPV testing. They concluded that cross-reactivity with nononcogenic HPV types had little effect on overall clinical performance. Alameda et al. [7] compared the performance of HC2 and PCR using GP5+/6+ primers in 83 ASCUS cases and found $25.3 \%$ cross-reactivity with low-risk HPV. They also emphasized the importance of HPV typing, especially for HPV 16 infection.

Age is another important parameter for defining the target population to undergo screening [8]. We found that women aged 30 years or older benefited more from HC2 screening. Furthermore, the association between persistence and high-grade cervical lesions is supposed to be more pronounced among women aged 25 years and older. Consequently, cervical screening works better in older women [8]. HPV infections tend to be transient, and even the majority of abnormalities in young women, including CIN2 and CIN3, tend to regress spontaneously, a scenario that may represent overtreatment and an increased risk of pregnancy-related morbidity [6]. There is no benefit to screening the youngest women, but there is a significant risk reduction for women aged 30-37 years. Also, women aged 20-24 years contribute little to the incidence of cervical cancer [8].

Regarding molecular tests, an HPV-positive status in young women can generate anxiety and psychological trauma when treating CIN that would never progress to cancer. For both situations, physicians can explain the implications of a positive test at a young age and minimize the adverse effects of overtreatment. Vaccination before exposure to HPV also can reduce these problems [9].

Because the screening goal is to avoid invasive cancer, it is indispensable to evaluate HPV testing performance. 
Katki et al. [10] studied the 5-year cumulative incidence of cervical cancer and CIN3+ in 331,818 women aged 30 years and older enroled in cotesting. They found a 5 -year cumulative incidence of cancer for HPV-negative women of 3.8 per 100,000 women, which was slightly higher than for the group undergoing both HPV testing and cytology (3.2 per 100,000 women). The rate for women with a negative Pap test result was 7.5 per 100,000 women. They also found that $73 \%$ of the women who were positive by HPV testing had no cytological abnormalities. Among these women, 35\% had CIN3 or adenocarcinoma in situ, 29\% had invasive carcinoma and $63 \%$ had invasive adenocarcinoma. The authors concluded that incorporating HPV testing with cytology also resulted in earlier identification of women at high-risk of cervical cancer, especially adenocarcinoma, and that HPV testing without adjunctive cytology might be sufficiently sensitive for primary screening. Dillner et al. [8] reported similar results. They evaluated 24,295 women and calculated the long-term cumulative incidence of CIN3+. The cumulative incidence rate of CIN3 + after 6 years for HPV-negative women was 0.27 versus $0.75 \%$ for women with negative cytology. Women with negative cytology results but who were HPV-positive after 6 years had a cumulative incidence rate of up to $10 \%$, while women who were HPV-negative but with cytological abnormalities had a $3 \%$ cumulative incidence rate of CIN3+.

The major advantage of including HC2 in routine evaluations can be the increase in the length of screening intervals; these can be safely lengthened to 6 years among women with a negative HPV test [6]. Women with normal cytology but a positive HPV test had a permanently increasing cumulative incidence rate of CIN3+, eventually reaching 10\% after 6 years. Women with an abnormal cytology and a negative HPV test had a cumulative incidence rate for CIN3+ of $2.7 \%$, and women with normal results for both tests had a very low risk of future CIN3+ $(0.28 \%)$.

Women with an HPV-positive test result and who are cytology-negative are believed to be safely managed by repeating both tests after 1 year. This option allows for increasing sensitivity for high-grade lesions in comparison to conventional cytology alone, with only a small increase in false-positives even in women younger than 35 years [11]. What is also advantageous is that the requirements for obtaining an adequate sample are less rigorous for HPV testing than for cytology [11].The overall relative sensitivity and specificity for self-taken samples is 74 and $84 \%$, respectively, suggesting that self-sampling for HPV testing is a valuable screening method for women who

HPV Testing in a Public Health Hospital decline or who do not have the opportunity for an appropriate clinician-based screening, and is thus an important way of improving population coverage of screening [11-13].

Cost estimates are an important issue for decisionmakers in public health systems. Screening alternatives rely on different levels and types of resources such as laboratory infrastructure, staff mix and clinical visits. In 2006, Goldhaber-Filbert and Goldie [14] reported estimated costs in 5 developing countries (India, Kenya, Peru, South Africa and Thailand) of cervical cytology and HPV testing. Cervical cytology is more labor-intensive, requiring a broader range and quantity of labor input with less reliance on equipment. HPV laboratories rely on automated processing, thus requiring less staff but requiring specific equipment. HPV tests are more expensive than cytology, and the higher sensitivity increases referrals to colposcopy, which makes HPV testing more expensive at first sight. But if we consider the long term, HC2 may be economically viable once it can be safely used at up to 6-year intervals among women with a negative result on the HPV test, while a single cervical cytology test is insensitive for the detection of precancer and cancer of the cervix.

Because of significant data confirming that HPV testing is substantially more sensitive than cytology at detecting CIN2+, some authors have suggested its use as primary screening. However, HPV testing is somewhat less specific than cytology, primarily because of the detection of transient infections that have not produced cytological changes. These facts raise questions such as 'Should every HPV-positive woman be referred for colposcopy?' If so, this strategy would become very costly. To answer these questions, Cuzick et al. [3] proposed a possible algorithm for the use of HPV testing as primary screening. Women aged 25-64 years old should be included. Women who test doubly negative could be returned to routine screening and be recalled after 5 years. If $\mathrm{HPV}$ testing is positive, cytology should be performed. Only if cytology is positive should women be referred for colposcopy. Management of HPV-positive and cytologynegative women presents a challenge, however. These women could be safely managed by repeating the testing with both cytology and HPV after 1 year.

Finally, our study has some evident biases. First, all patients referred for the gynecological examination had had a previous abnormal Pap test, follow-up of a treated cervical lesion, etc., which resulted in $39 \%$ of patients being HPV-positive, obviously higher than the average population. Patients with former cervical lesion treat- 
ment and those with immunosuppression were evaluated in different groups, to correct the bias. However, these groups were not statistically significant in the overall analysis. Another major bias was age. The FDAapproved HC2 for women older than 30 years and screening programs for women under the age of 25 years are not effective. Brazil's Health Ministry, however, recommends that the first Pap smears should be collected after the first sexual intercourse no matter a woman's age, which sometimes obliges practitioners to use HC2 for these young women very early. The recognition of the strong relationship between persistent infection with hrHPV types and the occurrence of cervical cancer has resulted in the development of a number of DNA and RNA detection systems for screening. Compared with cytology, HPV testing has a greater sensitivity [6], and the public health authorities could not ignore this important and strong evidence. In our study, HPV testing was more sensitive than cytology, and for women aged 30 years or older, the specificity was quite acceptable (70\%). Robust data published from around the world support the use of HPV testing for primary screening, and the combination with liquid-based cytology also offers an increased specificity [15]. Our study has demonstrated that women in Brazil could benefit from this combination.

\section{Acknowledgments}

The authors are indebted to Luciane Sarahyba and Nazareno dos Santos of the Molecular Biology Laboratory for their technical assistance.

\section{References}

1 Parkin DM, Whelan SL, Ferlay J, Raymind L, Young J (eds): Cancer Incidence in Five Continents, vol VII. Lyon, IARC Scientific Publications No. 143, 1997.

2 Castle PE, Rodrígues AC, Burk RD, Herrero R, Hildesheim A, et al: Neither one-time negative screening tests or negative colposcopy provides absolute reassurance against cervical cancer. Int J Cancer 2009;125:1649-1656.

3 Cuzick J, Arbyn M, Sankaranarayanan R, et al: Overview of human papillomavirusbased and other novel options for cervical cancer screening in developed and developing countries. Vaccine 2008;26S:K29-K41.

4 Bulkmans NWJ, Berkhof J, Rozendal L, et al: Human papillomavirus DNA testing for detection of cervical intraepithelial neoplasia grade 3 and cancer: 5 -year follow-up of a randomized controlled implementation trial. Lancet 2007;370:1764-1772.

5 Meijer C, Berkhof J, Castle PE, et al: Guidelines for human papillomavirus DNA test requirement for primary cervical cancer screening in women 30 years old and older. Int J Cancer 2009;124:516-520.
6 Ronco G, Rossi PG, Carozzi F, et al: Results at recruitment from a randomized controlled trial comparing human papillomavirus testing alone with conventional cytology as the primary cervical cancer screening test. JNCI 2008;100:492-501.

7 Alameda F, Bellosillo B, Lloveras B, Pairet S, Musset M, Pijuan L, Mariñoso L, Mancebo G, Larrazabal F, Carreras R, Serrano S: PCR study of a series of ASCUS cases HPV-positive by HCII. Diagn Cytopathol 2011, E-pub ahead of print.

8 Dillner J, Reboli M, Birembaut P, et al: Longterm predictive values of cytology and human papillomavirus testing in cervical cancer screening: joint European cohort study. BMJ 2008;377:a1754.

9 Sansieni P, Castanon A, Cuzick J, Snow J: Effectiveness of cervical screening with age: population-based case-control study of prospectively recorded data. BMJ 2009;339: b2968.

10 Katki HA, Kinney WK, Fetterman B, Lorey T, Poitras NE, Cheung L, Demuth F, Schiffman M, Wacholder S, Castle PE: Cervical cancer risk for women undergoing concurrent testing for human papillomavirus and cervical cytology: a population-based study in routine clinical practice. Lancet Oncol 2011;12:663-672.
11 Cuzick J, Mayrand MH, Rong G, Snijders P, Wardle J: Chapter 10: new dimensions in cervical cancer screening. Vaccine 2006; 24s:3/90-s3/97.

12 Ogilvie GS, Patrick DM, Schulzer M, et al: Diagnostic accuracy of self-collected vaginal specimens for human papillomavirus compared to clinician-collected human papillomavirus specimens: meta-analysis. Sex Transm Infect 2005;81:207-212.

13 Arbyn M, Sankaranarayanan R, Muwonge R, Keita N, Dolo A, Mbalawa, et al: Analysis of the accuracy of five cervical cancer screening tests assessed in eleven studies in Africa and India. Int J Cancer 2008; 123:153-160.

14 Goldhaber-Filbert J, Goldie S: Estimating the cost of cervical cancer screening in five developing countries. Cost Eff Resour Alloc 2006;4:13.

15 Schiffman M, Wentzensen N, Wacholder S, Kinney W, Gage JC, Castle PE: Human papillomavirus testing in the prevention of cervical cancer. J Natl Cancer Inst 2011;103: 368-383.

Beldi/Tacla/Caiaffa-Filho/Ab'saber/

Siqueira/Baracat/Alves/Longatto-Filho 